\title{
Moral Objectivism in Cross-Cultural Perspective
}

\author{
James Beebe
}

Department of Philosophy, State University of New York at Buffalo, 131 Park

Hall, Buffalo, NY 14260-4150, USA

Corresponding author, e-mail:jbeebe2@buffalo.edu

\section{Runya Qiaoan}

Sun Yat-sen University, Kanton, P.R. China

\section{Tomasz Wysocki}

Department of Philosophy, Washington University in St. Louis, One Brookings

Drive, St. Louis, Mo 63130-4899, USA

\section{Miguel A. Endara}

Azusa Pacific Online University, 901 E. Alosta Avenue, Azusa, CA 91702-7000, USA

\begin{abstract}
Moral psychologists have recently turned their attention to the study of folk metaethical beliefs. We report the results of a cross-cultural study using Chinese, Polish and Ecuadorian participants that seeks to advance this line of investigation. Individuals in all three demographic groups were observed to attribute objectivity to ethical statements in very similar patterns. Differences in participants' strength of opinion about an issue, the level of societal agreement or disagreement about an issue, and participants' age were found to significantly affect their inclination to view the truth of an ethical statement as a matter of objective fact. Implications for theorizing about folk morality are discussed.
\end{abstract}

\section{Keywords}

folk psychology - moral psychology - morality - objectivism - relativism 


\section{Introduction}

One of the most exciting areas of current research in moral psychology is the study of folk metaethical commitments (Goodwin and Darley, 2008; Sarkissian et al., 2011; Beebe and Sackris, data not shown; Wright et al., 2013). In contrast to what philosophers call 'normative ethical questions' about what it is that makes certain actions morally right or wrong, metaethical questions concern questions like the distinction between moral norms and social conventions, the possible variation in what morality requires from culture to culture or person to person, and the objectivity and truth conditions of moral judgments.

The impetus for much of the latest research on the relativist or objectivist views of ordinary individuals is a paper by Geoffrey Goodwin and John Darley (2008), in which they report that American undergraduates tend to regard moral judgments as more objective than statements of social conventions or preferences and almost as objective as statements of scientific fact. They also observed considerable variation across individuals and across different ethical issues. In a subsequent study, James Beebe and David Sackris (data not shown) found that American undergraduates attributed significantly less objectivity to moral judgments than did younger or older individuals from the same region of the United States. Hagop Sarkissian, John Park, David Tien, Jennifer Cole Wright, and Joshua Knobe (2011) reported that metaethical judgments tend to vary as a function of cultural distance, with increased distance (i.e., decreasing cultural similarity) leading to decreased attributions of metaethical objectivity. Jennifer Cole Wright, Piper Grandjean, and Cullen McWhite (2013) found that in addition to there being significant diversity among individuals with regard to the objectivity they attribute to moral judgments, there is also significant diversity of opinion with respect to whether certain issues are viewed as moral issues at all.

Together, these findings appear to undermine the traditional view among philosophers that ordinary people view moral judgments as uniformly and fully objective - i.e., as being as mind-independently true or false as the most uncontroversial scientific statements (cf., Mackie, 1977; Brink, 1989; Smith, 1994; Darwall, 1998 for statements of this view). While it will come as no surprise to social scientists that a sweeping claim like this (made without any attempt to ground it in scientific evidence) should turn out to be false, it nevertheless counts as news within the halls of professional philosophy.

With only two exceptions, all of the participants in the studies mentioned above have been American undergraduates. In one of their studies, Sarkissian et al. (2011) recruited undergraduates from Singapore, in order to show that the results they obtained with American students generalized to at least one 
other participant population. In addition, Beebe and Sackris (data not shown) recruited more than two thousand participants between the ages of 12 and 88 who were not college students. However, all of their participants either resided in western New York or had a connection to a large, public university located there. This means that the participant populations that have been studied so far have been rather limited in scope.

In an effort to extend the empirical investigation of folk metaethical commitments to a wider and more culturally diverse class of individuals, we deployed research materials analogous to those used by Beebe and Sackris (data not shown) in China, Poland, and Ecuador. We found many of the same basic patterns of responses observed by Beebe and Sackris. In Section 2, we describe the methods and key results of Beebe and Sackris and specify more precisely what we expected to observe in our participant populations. In Section 3, we report the results of our cross-cultural study. Finally, in Section 4, we briefly discuss the most important lessons or implications of our findings.

\section{Moral Objectivism across the (American) Lifespan}

Drawing inspiration from Goodwin and Darley (2008), Beebe and Sackris (data not shown) constructed the following list of statements about matters of physical fact, morality, and taste - including a roughly equal proportion of controversial and uncontroversial statements within each domain. Statements such as (2) and (7) - about global warming and the age of the Earth - are controversial within the United States, whereas statements (6) and (8) - about the smallest planet and the relative location of two well-known American cities - are not. Similarly, euthanasia (10) and abortion (12) are controversial within the U.S., but robbery (16) and assault (17) are uncontroversially seen as bad. Beebe and Sackris suspected that participants would also naturally distinguish between statements of taste that were controversial and those that were uncontroversial. For example, they hypothesized that statements comparing the musical merits of Beethoven and Britney Spears (23) and the attractiveness of two American celebrities whose handsomeness ratings were very far apart (21) would be viewed as significantly less controversial than other taste claims.

Beebe and Sackris (data not shown) directed participants to complete three tasks with respect to the statements in Figure 1. In Task 1, participants indicated the degree to which they agreed or disagreed with each statement on a six-point scale from ' 1 ' ('Strongly Disagree') to '6' ('Strongly Agree'). In Task 2, participants were asked the following question: 


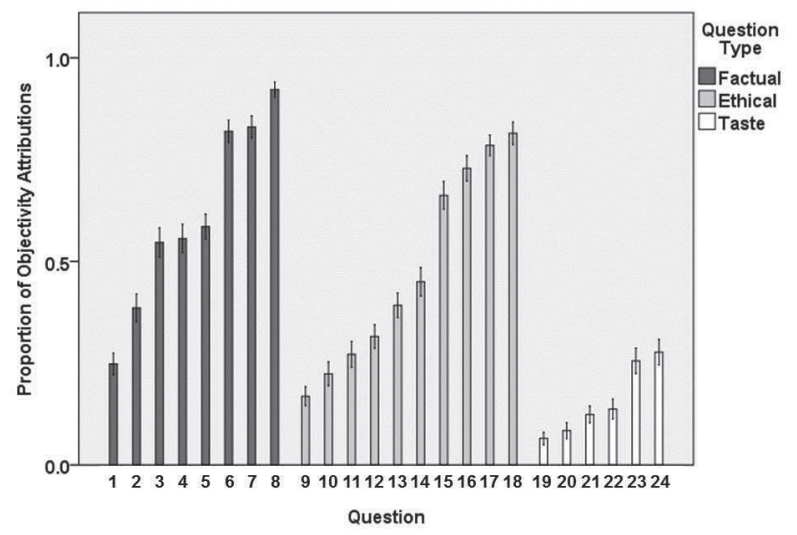

FIGURE 1 Proportions of participant objectivity attributions to the factual, ethical, and taste statements that appear in Table 1. Error bars in all figures represent $95 \%$ confidence intervals.

If someone disagrees with you about whether (one of the test statements is true), is it possible for both of you to be correct or must one of you be mistaken?

(A) It is possible for both of you to be correct.

(B) At least one of you must be mistaken.

Beebe and Sackris interpreted (B) as an ascription of objectivity to the statement in question and (A) as a denial of objectivity. While there are likely to be multiple dimensions or kinds of objectivity that can be attributed to a moral statement, the Task 2 question seems to capture one of the most important dimensions. If some version of subjectivism is true, according to which one's beliefs, decisions, preferences, or other mental states make moral statements true or false, it may be impossible to be mistaken about them. Alternatively, if some version of emotivism is true (e.g., Ayer 1936, Stevenson 1946), and moral statements have no genuine truth conditions, two disagreeing parties would again be shielded from the possibility of being mistaken. Task 2 attempts to measure whether individuals take moral statements to have mind-independent truth conditions. In Task 3 , Beebe and Sackris directed participants to indicate the extent to which they thought that "people in our (i.e., American) society" disagreed about the statements in question. Participants registered their opinions on a six-point scale that ranged from 'There is no disagreement at all' to 'There is an extremely large amount of disagreement.' 
The proportions of objectivist responses that Beebe and Sackris observed in their study are represented in Figure 1.

The average proportion of objectivity attributions was 0.60 for factual claims, 0.47 for ethical claims, and 0.15 for taste claims. The difference in the proportions of objectivity attributions to factual and ethical statements constituted a small effect size (Cramér's $V=0.12$ ), while the difference between objectivity attributions to ethical and taste claims represented a medium effect size (Cramér's $V=0.33$ ). Most philosophers would have predicted significantly higher objectivity attributions for both factual and ethical claims. As can be seen in Figure 1, there was also significant variation in the proportion of objectivity responses within each subcategory, with some ethical claims being treated as more objective than some factual claims, and some taste claims treated as more objective than some ethical claims. Participants on the whole had stronger opinions about ethical statements than about factual ones (when Beebe and Sackris recoded all (strong) ' 1 ' and ' 6 ' responses on Task 1 as ' 3 ,' all (moderate) ' 2 ' and ' 5 ' responses as '2,' and (weak) ' 3 ' and ' 4 ' responses as '1,' the mean strength of opinion rating was 2.20 for factual statements, 2.45 for ethical statements, and 2.22 for taste claims. The greater strength of belief in regard to ethical statements represented a small effect size (Cramér's $V=0.10)$.) and perceived greater disagreement about ethical claims (3.58) than factual (3.29) or taste (3.31) claims (this represents a small effect size (Cramér's $V=0.12)$ ). Beebe and Sackris also found that, while participants' attributions of objectivity were positively correlated with the strength of their opinions (factual: $r_{\mathrm{pb}}=0.26$; ethical: $r_{\mathrm{pb}}=0.41$; taste: $\left.r_{\mathrm{pb}}=0.24\right)$ and negatively correlated with the extent to which they thought there was disagreement within society about the issue (factual: $r_{\mathrm{pb}}=-0.17$; ethical: $r_{\mathrm{pb}}=-0.26$; taste: $r_{\mathrm{pb}}=-0.13$ ), participants showed an ability to distinguish the issues of strength of agreement, perceived disagreement, and objectivity.

The most significant result obtained by Beebe and Sackris concerns the association between participants' age and their inclination to attribute objectivity to ethical statements. The average proportion of objectivity attributions in the 17 to 29 year old participant group (0.37) was significantly lower than the averages in the 12 to $16(0.47), 30$ to $51(0.56)$, or $5^{2}$ to 88 (0.48) groups (17 - 29 vs. $12-16: \chi^{2}(1, N=1849)=19.431, p<0.001$, Cramér's $V=0.10$ (small effect size). $17-29$ vs. $30-51: \chi^{2}(1, N=1962)=68.974, p<0.001$, Cramér's $V=0.19$ (small effect size). $17-29$ vs. $5^{2}-88: \chi^{2}(1, N=6921)=46.235, p<0.001$, Cramér's $V=$ 0.08 (small effect size)). The best explanation for these differences seems to be that it is during their late teens and early twenties that individuals are most open to new experiences and most actively engaged in self-exploration and 
identity-formation and that this psychic fluidity and openness leads them to be receptive to the possibility of there being more than one correct moral system (McCrae \& Costa, 2003; Srivastava, John, Gosling \& Potter, 2003).

In our study, we wanted to investigate the extent to which similar results might be obtained in different cultural settings. We formulated the following hypotheses about what we would observe:

(H1) Individuals in China, Poland, and Ecuador would attribute more objectivity to factual claims than to ethical claims and more objectivity to ethical claims than to taste claims.

$(\mathrm{H} 2)$ Within each participant group, the average amount of objectivity attributed to ethical statements would fall far below the level predicted by traditional philosophical theories.

$\left(\mathrm{H}_{3}\right)$ Within each participant group, there would be considerable variation in individuals' willingness to attribute objectivity to ethical statements.

$\left(\mathrm{H}_{4}\right)$ Within each participant group, some ethical claims would be treated as more objective than some factual claims, and some taste claims treated as more objective than some ethical claims.

$\left(\mathrm{H}_{5}\right)$ For each statement type, the attributions of objectivity within each participant group would be positively associated with participants' strength of opinion about the matter.

(H6) For each statement type, the attributions of objectivity of each participant group would be negatively associated with the extent to which participants thought there was disagreement within their society about the statements.

$\left(\mathrm{H}_{7}\right)$ Within each participant group, an age effect would be observed, where individuals in their late teens and twenties would attribute less objectivity to ethical claims than individuals in younger or older age groups.

In short, we predicted that the major findings of Beebe and Sackris (data not shown) would on the whole be replicated in different cultures, thereby revealing widely shared features of folk metaethical commitments.

The materials that we used were selected from those used by Goodwin and Darley (2008) and Beebe and Sackris (data not shown) and are represented in Table 1. 
TABLE 1 Factual, ethical and taste claims used in the study by Beebe and Sackris (data not shown)

Factual

1. Frequent exercise usually helps people to lose weight.

2. Global warming is due primarily to human activity (for example, the burning of fossil fuels).

3. Julius Caesar did not drink wine on his 21st birthday.

4. There is an even number of stars in the universe.

5. Humans evolved from more primitive primate species.

6. Mars is the smallest planet in the solar system.

7. The earth is only 6ooo years old.

8. New York City is further north than Los Angeles.

Ethical

9. Anonymously donating a significant portion of one's income to charity is morally good.

10. Assisting in the death of a friend who has a disease for which there is no known cure and who is in terrible pain and wants to die is morally permissible.

11. Scientific research on human embryonic stem cells is morally wrong.

12. Before the third month of pregnancy, abortion for any reason is morally permissible.

13. Cutting the American flag into pieces and using it to clean one's bathroom is morally wrong.

14. Lying on behalf of a friend who is accused of murder is morally permissible.

15. Cheating on an exam that you have to pass in order to graduate is morally permissible.

16. Robbing a bank in order to pay for an expensive vacation is morally bad

17. Hitting someone just because you feel like it is wrong.

18. Treating someone poorly on the basis of their race is morally wrong.

Taste

19. Classical music is better than rock music.

20. McDonald's hamburgers taste better than hamburgers made at home.

21. Brad Pitt is better looking than Drew Carey.

22. Gourmet meals from fancy Italian restaurants taste better than microwavable frozen dinners.

23. Beethoven was a better musician than Britney Spears is.

24. Barack Obama is a better public speaker than George W. Bush.

Brad Pitt is an American actor known for his good looks, and Drew Carey is an American comedian and television personality who is known for his quirky looks that few would describe as handsome. 
Native Chinese, Polish, and Spanish speakers translated these statements into their respective languages, and then independent translators translated them back into English in order to test for accuracy. Any discrepancies were resolved through collaborative discussion. Statements were tailored to make them culturally relevant, when necessary.

Each of the Chinese, Polish, and Spanish statement sets was broken up into three different questionnaires. Participants completed the same Tasks 1 through 3 described above for the each of the statements in their questionnaire. Although Goodwin and Darley (2008) and Beebe and Sackris (data not shown) both employed six-point answer scales for their participants to use in Tasks 1 and 3, we thought it was preferable to use five-point scales, so that participants who had no inclination either to agree or to disagree with a test item would have a neutral mid-point to use.

The participants from China were 414 native Chinese speakers $(46 \%$ female) between the ages of 11 and 80 residing in major metropolitan cities in the Henan and Shandong provinces. Polish participants were 299 native Polish speakers ( $55 \%$ female) between the ages of 19 and 82 living in Wroclaw in southwestern Poland. In Ecuador, there were 180 native Spanish speakers (59\% female) between the ages of 18 and 81 living in the capital city of Quito. All participants were drawn from large, metropolitan areas. Only in China were there any participants under the age of 18.

The proportion of objectivity attributions that participants from China, Poland, and Ecuador made to the statements in Table 2 are represented in Table 3 . The data represented in Table 3 and Figure 2 include all participants, including those from China who were under the age of 18. Limiting the data only to adults from the three countries had no appreciable effect and so all data were included in the subsequent analysis. The statements in both tables are organized according to increasing proportions of objectivity attributed to them. Like the American participants in Beebe and Sackris' study, participants in all three demographic groups were much more inclined to attribute objectivity to statements about Mars and geographical locations than to statements about exercise and unknowable truths from the distant past.

We followed Beebe and Sackris (data not shown) and included a factual item whose truth value was not only unknown but practically unknowable (claim (2) in Table 2), in order to make a point about the possible limitations of our test for objectivity and to suggest directions for future study. We find it difficult to believe that anyone could clearheadedly believe that there is no fact of the matter about whether Confucius (or Julius Caesar) did or did not eat soup on his twenty-first birthday. Yet participants in our study (as in Beebe and Sackris') were rather reluctant to choose the 'At least one of two 
Factual

1. Frequent exercise usually helps people to lose weight.

2. Confucius (or some other ancient cultural figure) did not eat soup on his 21st birthday.

3. New York City (or some other city or country) is further north than Los Angeles (another city or country).

4. Mars is the smallest planet in the solar system.

\section{Ethical}

5. Anonymously donating a significant portion of one's income to charity is morally good.

6. Assisting in the death of a friend who has a disease for which there is no known cure and who is in terrible pain and wants to die is morally permissible.

7. Lying on behalf of a friend who is accused of murder is morally permissible.

8. Cheating on an exam that you have to pass in order to graduate (or obtain a job) is morally permissible.

9. Cutting the Chinese (or Polish or Ecuadorian) flag into pieces and using it to cleattn one's bathroom is morally wrong.

10. Treating someone poorly on the basis of their race is morally wrong.

11. Hitting someone just because you feel like it is wrong.

Taste

12. Mozart was a better musician than Michael Jackson.

13. Traditional Chinese (or Polish or Ecuadorian) music is better than rock music.

14. Meals from an upscale restaurant taste better than food from McDonald's.

15. The Great Wall of China (or some well-known public figure) is more beautiful than the Three Gorges Dam (another public figure).

Each participant group also responded to three additional statements, but these do not appear in Table 2 because they were not uniform across the three groups. The full text of the questionnaires can be found at (permanent url). With regard to claim No. 15, one concern about our research materials stems from the fact that our factual and ethical statements were non-comparative, asking participants to consider one object or event at a time, while our taste claims were comparative, asking participants to consider pairs of items. Any significant difference we obtain between participant assessments of these statement categories could well be due to differences of formulation rather than the core features of the statement categories themselves. Cova and Pain (2012), however, recently completed a study of the objectivity attributions of ordinary participants made to aesthetic statements. Using non-comparative statements that included the French predicates 'beau' (beautiful) or 'laid' (ugly), they obtained results equivalent to the ones we report below. Thanks to $\mathrm{cK}$ for pressing this point with us. 
TABLE 3 Proportions of objectivity attributions to the factual, ethical, and taste claims used in the present study, organized by participant group

\begin{tabular}{llllll}
\hline & & China & Poland & Ecuador & $M$ \\
\hline Factual & Benefits of exercise & 0.38 & 0.43 & 0.40 & 0.40 \\
& 21st birthday & 0.47 & 0.52 & 0.47 & 0.49 \\
& Geographical location & 0.71 & 0.74 & 0.72 & 0.72 \\
& Mars & 0.66 & 0.84 & 0.73 & 0.74 \\
Ethical & Donating money & 0.29 & 0.27 & 0.42 & 0.33 \\
& Euthanasia & 0.32 & 0.40 & 0.65 & 0.46 \\
& Lying & 0.53 & 0.48 & 0.62 & 0.54 \\
& Cheating & 0.59 & 0.71 & 0.57 & 0.62 \\
& Flag & 0.64 & 0.58 & 0.69 & 0.64 \\
& Racism & 0.52 & 0.63 & 0.77 & 0.64 \\
& Hitting & 0.55 & 0.78 & 0.68 & 0.67 \\
& Mozart vs. Jackson & 0.23 & 0.19 & 0.30 & 0.24 \\
& Traditional vs. rock music & 0.28 & 0.14 & 0.38 & 0.27 \\
& Fine food vs. McDonald's & 0.32 & 0.19 & 0.43 & 0.31 \\
& Beauty & 0.30 & 0.26 & 0.48 & 0.35 \\
& & & & & \\
\hline
\end{tabular}

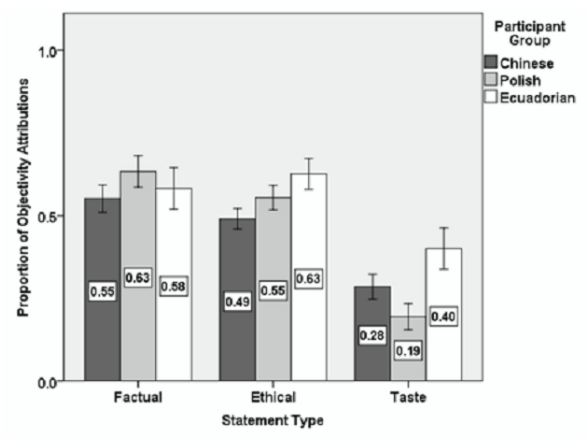

FIGURE 2

Proportions of participant objectivity attributions to factual, ethical, and taste claims, organized by participants' demographic group.

disagreeing must be wrong' answer in regard to this statement. Although we could be mistaken, we think it is much more likely that participants are confusing metaphysical and epistemological issues than that they genuinely have doubts about the existence of there being a mind-independent fact here. It takes a bit of cognitive or philosophical sophistication to recognize that the truth value of a statement is one thing, and our knowledge of it is another. 
Thus, we believe that at least with regard to this statement, participants were responding to something other than what we wanted them to be responding to, when they were completing Task 2 . While we are more open than some philosophers (e.g., Nichols 2004) to the possibility that ordinary individuals may well be non-realists or non-objectivists about basic factual matters, we think further investigation of folk intuitions about these matters is called for. We should note that, despite the fact that we have pointed to a possible limitation of the objectivity test we employed, we agree with Beebe and Sackris (data not shown) that it is superior to the other tools that have been employed in this area of research (cf., Beebe and Sackris (data not shown) for extended critical discussion of other measures that have been used).

Participants in all three of our demographic groups were much more likely to view the wrongness of racism and hitting other people as being objective matters than the goodness of donating to charity or the permissibility of euthanasia. Goodwin and Darley $(2008,2012)$ and Beebe and Sackris (data not shown) obtained similar results. The cross-cultural replication of the finding that the goodness of donating money to charity was uniformly viewed as being less objective than statements about the wrongness of certain moral violations suggests that these results are not due to any accidental differences of meaning between the pairs of moral terms 'good'/'bad' and 'right'/'wrong.' Rather, the data points toward there being some widely recognized distinction between different moral categories, with the actions in some categories viewed as weightier or more serious than others.

The average proportion of objectivity attributions to factual, ethical, and taste claims within each participant group are represented in Figure 2. Among Chinese and Polish participants, significantly more objectivity was attributed to factual claims than to ethical claims (Chinese: $\chi^{2}(1, N=1,523)=5.366, p<$ 0.05, Cramér's $V=0.06$ (small effect size). Polish: $\chi^{2}(1, N=1,089)=6.447, p<$ 0.05 , Cramér's $V=0.08$ (small effect size)). However, participants from Ecuador attributed more objectivity to ethical claims than to factual claims, although this difference failed to be statistically significant. Participants in all three demographic groups were significantly more inclined to attribute objectivity to ethical claims than to taste claims (Chinese: $\chi^{2}(1, N=1,523)=61.665, p<$ 0.001, Cramér's $V=0.20$ (small effect size). Polish: $\chi^{2}(1, N=1,088)=133.551$, $p<0.001$, Cramér's $V=0.35$ (medium effect size). Ecuadorian: $\chi^{2}(1, N=657)=$ 31.329, $p<0.001$, Cramér's $V=0.22$ (small effect size)).

The differences between the proportions of objectivity attributed to factual statements by Chinese (0.55), Polish (0.63), and Ecuadorian (0.58) participants were significant (factual: $\chi^{2}(2, N=1192)=6.409, p<0.05$, Cramér's $V=$ 0.07 (small effect size)). The between-group differences in the proportions of objectivity attributed to ethical and taste claims were also significant (ethical: 
$\chi^{2}(2, N=2,074)=22.597, p<0.001$, Cramér's $V=0.10$ (small effect size); taste: $\chi^{2}(2, N=1,194)=31.739, p<0.001$, Cramér's $V=0.16$ (small effect size)).

Looking at each participant group and statement category pair, objectivity attributions were for the most part positively associated with participants' strength of opinion about an issue, with the largest effects being found for ethical claims. The association between strength of belief and objectivity attributions among Chinese participants responding to factual claims: $\chi^{2}(2, N=562)=$ 30.163, $p<0.001$, Cramér's $V=0.23$ (small effect size). Chinese and ethical claims: $\chi^{2}(2, N=960)=115.295, p<0.001$, Cramér's $V=0.35$ (medium effect size). Chinese and taste claims: $\chi^{2}(2, N=562)=22.230, p<0.001$, Cramér's $V=0.20$ (small effect size). Polish and factual claims: $\chi^{2}(2, N=390)=32.780$, $p<0.001$, Cramér's $V=0.29$ (small to medium effect size). Polish and ethical claims: $\chi^{2}(2, N=695)=86.759, p<0.001$, Cramér's $V=0.35$ (medium effect size). Polish and taste claims: $\chi^{2}(2, N=391)=16.682, p<0.001$, Cramér's $V=$ 0.21 (small effect size). Ecuadorians and factual claims: $\chi^{2}(2, N=236)=5.917$, $p=0.05^{2}$, Cramér's $V=0.16$ (small effect size). Ecuadorians and ethical claims: $\chi^{2}(2, N=417)=23.858, p<0.001$, Cramér's $V=0.24$ (small effect size). Ecuadorians and taste claims: $\chi^{2}(2, N=240)=0.049, p=0.98$, Cramér's $V=0.01$. In other words, the stronger individuals' opinions were about a claim, the more likely they were to think there was an objective fact of the matter. The only exceptions to this pattern concerned Ecuadorians and factual claims, where the relationship was marginally significant, and Ecuadorians and taste claims, where there was no significant relationship.

Among Chinese and Polish individuals, attributions of objectivity were negatively associated with the extent to which they thought there was disagreement within their society about the target statements. The association between perceived disagreement and objectivity attributions among Chinese participants responding to factual claims: $\chi^{2}(4, N=562)=52.823, p<0.001$, Cramér's $V=0.31$ (medium effect size). Chinese and ethical claims: $\chi^{2}(4, N=$ $961)=105.449, p<0.001$, Cramér's $V=0.33$ (medium effect size). Chinese and taste claims: $\chi^{2}(4, N=562)=29.782, p<0.001$, Cramér's $V=0.23$ (small effect size). Polish and factual claims: $\chi^{2}(4, N=393)=35.128, p<0.001$, Cramér's $\mathrm{V}=$ 0.30 (medium effect size). Polish and ethical claims: $\chi^{2}(4, N=696)=38.372$, $p<0.001$, Cramér's $V=0.24$ (small effect size). Polish and taste claims: $\chi^{2}$ (4, $N=390)=25.713, p<0.001$, Cramér's $V=0.26$ (small effect size). However, there was no such association among Ecuadorian participants. In other words, Chinese and Polish participants were less likely to attribute objectivity to statements that they thought were widely disputed and were more likely to attribute objectivity to statements about settled matters.

Strikingly, participants in their late teens and twenties were significantly less likely to attribute objectivity to ethical statements in all three participant 


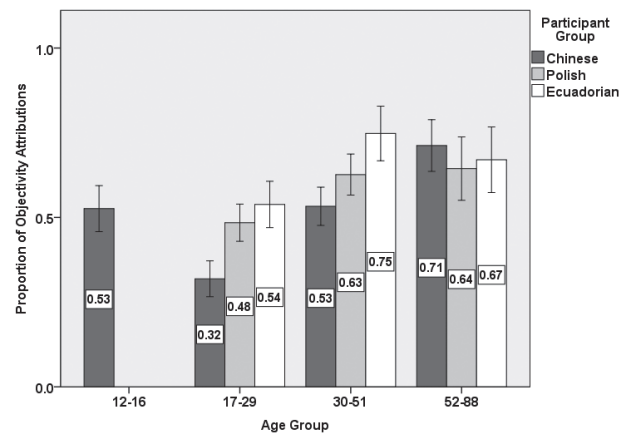

FIGURE 3

Proportions of participant objectivity attributions to ethical claims, organized by participant age and demographic group.

groups - just as Beebe and Sackris (data not shown) observed in their sample of Americans (cf., Figure 3) (Chinese: $\chi^{2}(3, N=955)=66.052, p<0.001$, Cramér's $V=$ 0.26 (small effect size). Polish: $\chi^{2}(2, N=675)=14.924, p<0.01$, Cramér's $V=0.15$ (small effect size). Ecuadorian: $\chi^{2}(2, N=417)=14.881, p<0.01$, Cramér's $V=0.19$ (small effect size)).

In none of the demographic groups were there significant differences in how participants from different age groups viewed the objectivity of factual claims. Chinese and Polish participants aged 17 to 29 were significantly less likely to attribute objectivity to taste claims than were other individuals from these samples (Chinese: $\chi^{2}(3, N=557)=30.037, p<0.001$, Cramér's $V=0.23$ (small effect size). Polish: $\chi^{2}(2, N=379)=12.009, p<0.01$, Cramér's $V=0.18$ (small effect size)). This result contrasts with Beebe and Sackris' (data not shown) finding that the objectivity attributed by American participants to taste claims decreased with age. The only gender-based difference in objectivity attributions was that females in China attributed less objectivity to ethical claims (o.43) than males $(0.54)\left(\chi^{2}(1, N=961)=9.819, p<0.01\right.$, Cramér's $V=0.10$, small effect size).

Thus, almost every aspect of the hypotheses $\left(\mathrm{H}_{1}\right)$ through $\left(\mathrm{H}_{7}\right)$ above was confirmed by our study. Participants in China and Poland (but not Ecuador) attributed more objectivity to factual claims than ethical claims, and participants in all three groups attributed more objectivity to ethical claims than taste claims $\left(\mathrm{H}_{1}\right)$. The average amount of objectivity attributed to ethical statements fell significantly below what traditional philosophical theories would predict ( $\left.\mathrm{H}_{2}\right)$. On the whole, participants in each demographic group rarely approached unanimity in their objectivity attributions, displaying considerable intra-cultural variation $\left(\mathrm{H}_{3}\right)$. Within each participant group, some ethical claims were treated as more objective than some factual claims, and some taste claims were treated as more objective than some ethical claims $\left(\mathrm{H}_{4}\right)$. Attributions of objectivity were in general positively associated with the 
strength of participants' opinions but negatively associated with the extent to which they thought there was societal disagreement about the statements $\left(\mathrm{H}_{5}\right.$ and H6). Finally, individuals in their late teens and twenties from all cultures attributed less objectivity to ethical claims than individuals in younger or older age groups $\left(\mathrm{H}_{7}\right)$.

\section{Discussion}

One important lesson from our data is that individuals from at least some different parts of the globe agree in treating moral statements as considerably more objective than taste claims. While some philosophers have occasionally defended subjectivist visions of morality (e.g., Protagoras, Hume), very few (if any) have ever maintained that this is how the ordinary person in the street views morality. The norm among moral theorists has been to maintain that ordinary individuals view morality as being strongly objective. J.L. Mackie $(1977,33)$, for example, claimed:

The ordinary user of moral language means to say something about whatever it is that he characterizes morally, for example a possible action, as it is in itself... and not about, or even simply expressive of, his, or anyone else's, attitude or relation to it... one that is absolute, not contingent upon any desire or preference or policy or choice.

Michael Smith $(1994,6,84)$ has argued that "we seem to think moral questions have correct answers; that the correct answers are made correct by objective moral facts." and that "it is a platitude that our moral judgements at least purport to be objective"). Indeed, what Smith calls 'the moral problem' is constituted by the following conjunction of alleged facts that do not sit well together: (i) Moral Objectivity: moral judgements are beliefs about matters of fact, (ii) Internalism: moral judgements, by themselves, motivate those who make them, and (iii) Humean Theory of Motivation: beliefs and desires are distinct states; beliefs on their own cannot motivate. Similar claims have been made by David Brink (1989) and Stephen Darwall (1998) - and tacitly taken for granted by countless others. However, the variation that we observed across issues and across individuals (and that Goodwin and Darley 2008 and Beebe and Sackris observed as well) suggest that the folk conception of morality is not as uniformly and strongly objective as these philosophers have assumed.

Another important lesson from our study concerns the fact that the objectivity attributions of participants in each of our samples and those in Beebe and Sackris' study were affected in the same ways by the same factors. When an issue was viewed as being settled or uncontroversial, participants were much 
more likely to attribute objectivity to a statement about it. But when an issue was viewed as being highly contentious or unsettled, participants inclined toward non-objectivist responses. Recent experimental manipulations by Beebe (2014) and Goodwin and Darley (2012) show that causal conclusions can be drawn about the negative association between metaethical objectivity attributions and perceived societal disagreement. When the extent of disagreement about an issue was made salient and its degree was manipulated, objectivity attributions decreased.

Although we observed a significant degree of cross-culturally uniformity in our study, we do not wish to draw overly strong conclusions about the universality of folk metaethical commitments from our data. From the recent history of moral psychology, we are aware that the initial evidence seemed to support Elliot Turiel's (1983) claims about the universality of the moral/conventional distinction but that later evidence emerged to reveal significant cross-cultural diversity (e.g., Shweder, Mahapatra \& Miller 1987; Haidt, Koller \& Dias 1993). We wish to draw the modest conclusion that in regard to the hypotheses laid out in $\left(\mathrm{H}_{1}\right)$ through $\left(\mathrm{H}_{7}\right)$, there was significant uniformity in our three participant populations. We believe that the best way to advance the moral-psychological study of folk metaethical commitments is to study them from a non-WEIRD perspective (Henrich et al. 2010), and thus we hope that our results will contribute to a better understanding of folk thinking in this domain.

\section{References}

Ayer, A.J. (1936). Language, Truth and Logic. Victor Gollancz, London.

Beebe, J.R. (2014). How different kinds of disagreement impact folk metaethical judgments. In Cole Wright, J. and Sarkissian, H. (Eds.), Advances in Experimental Moral Psychology: Affect, Character, and Commitments, pp. 167-187. Continuum, London.

Beebe, J.R. and Sackris, D. (data not shown). Moral objectivism across the lifespan.

Brink, D.O. (1989). Moral Realism and the Foundations of Ethics. Cambridge University Press, Cambridge.

Cova, F. and Pain, N. (2012). Can folk aesthetics ground aesthetic realism? The Monist 95, 243-63.

Darwall, S. (1998). Philosophical Ethics. Westview Press, Boulder, co.

Goodwin, G.P. and Darley, J.M. (2008). The psychology of meta-ethics: exploring objectivism. Cognition 106, 1339-1366.

- (2012). Why are some moral beliefs perceived to be more objective than others? Journal of Experimental Social Psychology 48, 250-256. 
Haidt, J., Koller, S. and Dias, M. (1993). Affect, culture, and morality, or is it wrong to eat your dog? Journal of Personality and Social Psychology 65, 613-628.

Henrich, J., Heine, S.J. and Norenzayan, A. (2010). The weirdest people in the world? Behavioral and Brain Sciences 33, 61-83.

Mackie, J.L. (1977). Ethics: Inventing Right and Wrong. Penguin, London.

McCrae, R.R. and Costa Jr., P.T. (2003). Personality in Adulthood: A Five-Factor Theory Perspective, 2nd edn. Guilford Press, New York, NY.

Nichols, S. (2004). After objectivity: An empirical study of moral judgment. Philosophical Psychology 17, 3-26.

Sarkissian, H., Parks, J., Tien, D., Wright, J.C. and Knobe. J. (2011). Folk moral relativism. Mind \& Language 26, 482-505.

Shweder, R.A., Mahapatra, M. and Miller, J. (1987). Culture and moral development. In Kagan, J. and Lamb, S. (Eds.), The Emergence of Morality in Young Children, pp. 1-83. University of Chicago Press, Chicago, IL.

Smith, M. (1994). The Moral Problem. Blackwell, Oxford.

Srivastava, S., John, O.P., Gosling, S.D. and Potter, J. (2003). Development of personality in early and middle adulthood: set like plaster or persistent change? Journal of Personality and Social Psychology 84, 1041-1053.

Stevenson, C.L. (1937). Ethics and Language. Yale University Press, New Haven, Cт.

Turiel, E. (1983). The Development of Social Knowledge: Morality and Convention. Cambridge University Press, Cambridge.

Wright, J.C., Grandjean, P.T. and McWhite, C.B. (2013). The meta-ethical grounding of our moral beliefs: Evidence for meta-ethical pluralism. Philosophical Psychology 26, 336-361. 